\title{
Cianoacrilato na colagem de Bráquetes ortodônticos em resina acrílica: há maior adesão?
}

\author{
Bonding orthodontic braces in acrylic \\ resin with cyanoacrylate:does it improve \\ adesion?
}

\author{
Ayla Macyelle de Oliveira Correia ${ }^{1}$, Renata Pilli Jóias ${ }^{2}$, \\ Sigmar de Mello Rode ${ }^{2}$, Carlos Eduardo Repeke ${ }^{3}$, \\ Adriano Augusto de Melo Mendonça', Luiz Renato Paranhos ${ }^{3}$
}

\footnotetext{
${ }^{1}$ Departamento de Odontologia, UFS - Universidade Federal de Sergipe, CEP: 49060-100, Aracaju, SE. e-mail: aylamacyelle@hotmail.com; adri_amm@yahoo.com.br

${ }^{2}$ Departamento de Materiais Odontológicos e Prótese, UNESP - Universidade Estadual Paulista Júlio de Mesquita Filho, CEP: 12245-000, São José dos Campos, SP.

e-mail: pillijoias_renata@yahoo.com.br; sigmar@fosjc.unesp.br

${ }^{3}$ Departamento de Odontologia, UFS - Universidade Federal de Sergipe, CEP.: 49400-000 Lagarto, SE. e-mail: repeke@ufs.br; paranhos@ortodontista.com.br
}

\begin{abstract}
RESUMO
Pacientes em tratamento ortodôntico apresentam restaurações provisórias com frequência. No entanto, poucos estudos avaliam a influência dos adesivos na resistência ao cisalhamento dos bráquetes nessas superfícies. A resina acrílica é comumente indicada para colagem de bráquetes, porém o uso do cianoacrilato como adesivo ortodôntico é uma opção analisada. O objetivo do presente trabalho foi avaliar o desempenho do cianoacrilato associado aos materiais comumente utilizados para a fixação de bráquetes metálicos em restaurações provisórias de resina acrílica. Quarenta amostras em resina acrílica foram preparadas e as superfícies homogeinizadas com lixas de carboneto de silício (320 e 600). Em seguida, as amostras foram divididas aleatoriamente em quatro grupos $(n=10)$ com base no tratamento de superfície e agente de união: G1 - bráquetes colados com resina acrílica; G2 - bráquetes colados com resina acrílica e aplicação de cianoacrilato; G3 - bráquetes colados com Transbond ${ }^{\mathrm{TM}}$ XT; G4 - bráquetes colados com Transbond ${ }^{\mathrm{TM}}$ XT e aplicação de cianoacrilato. Foram utilizados bráquetes ortodônticos de aço inoxidável, prescrição Roth, Kirium (3M/Abzil) para incisivos centrais superiores direitos, slot 022. Após colagem, as amostras foram submetidas ao teste de cisalhamento a uma velocidade de $0,5 \mathrm{~mm} / \mathrm{min}$ em uma máquina de ensaios universal (EMIC DL-1000). Os dados foram coletados e submetidos à análise estatística pelo teste ANOVA com nível de significância de 5\%. A associação de resina acrílica ao cianoacrilato (G2) resultou na maior resistência ao cisalhamento (13,76 MPa), mas não significativa em comparação aos valores obtidos para a resina acrílica (G1= 7,76 MPa). O mesmo pôde ser observado para a associação Transbond ${ }^{\mathrm{TM}}$ XT e cianoacrilato (G4=4,03 $\mathrm{MPa}$ ) em relação a utilização da Transbond ${ }^{\mathrm{TM}} \mathrm{XT}$ de forma isolada (G3= 3,87 $\mathrm{MPa}$ ) e resina acrílica. O tratamento de superfície tem efeito significativo na resistência da união dos bráquetes colados aos materiais provisórios. A associação de cianoacrilato ao monômero de metilmetacrilato apresentou maior resistência ao cisalhamento, sendo mais indicada clinicamente.
\end{abstract}

Palavras-chave: Ortodontia. Resistência ao Cisalhamento. Colagem Dentária. Resinas Compostas. Resina Acrílica.

\section{ABSTRACT}

Patients in orthodontic treatments frequently present composite restorations. However, few studies evaluated the influence of bonding materials within braces shearing bond strength on composite surfaces. The acrylic 
resin became the recommended material for bonding brackets on these surfaces; however the use of cyanoacrylate emerged as a potential option. The present study aimed to evaluate the performance of cyanoacrylate associated with orthodontic materials commonly used for the fixation of metallic braces on temporary restorations of acrylic resin. Forty samples were prepared with acrylic resin and homogenized with silicon carbide sand paper (320 and 600). The samples were randomly divided in four groups $(\mathrm{n}=10)$ based on the material treatment and bonding agent: G1 - braces bonded with acrylic resin; G2 - braces bonded with acrylic resin combined with application of cyanoacrylate; G3 - braces bonded with Transbond ${ }^{\mathrm{TM}}$ XT; G4 braces bonded with Transbond ${ }^{\mathrm{TM}} \mathrm{XT}$ combined with application of cyanoacrylate. Only stainless steel braces were used, prescription Roth, Kirium (3M/Abzil) designed for maxillary right central incisors, slot 022. After bonding, the sample underwent shearing tests at $0.5 \mathrm{~mm} / \mathrm{min}$ within a standard testing device (EMIC DL1000). Statistical data consisted of ANOVA with significance level set at 5\%. Group 2 (G2) reached major shearing strength (13.76 MPa). However, it was not statistically significant in face of the outcomes of Group 1 (G1) (G1= 7.76 MPa). The same was observed for the association of Transbond ${ }^{\mathrm{TM}} \mathrm{XT}$ and cyanoacrylate (G4= 4.03MPa) compared with isolated Transbond ${ }^{\mathrm{TM}}$ XT $(\mathrm{G} 3=3.87 \mathrm{MPa})$ and acrylic resin. Surface treatment of orthodontic material revealed a significant effect on shearing strength of temporary materials. The association between cyanoacrylate with monomer of methyl methacrylate presented major shearing strength, becoming more indicated for clinical interventions.

Keywords: Orthodontics. Shear Bond Strength. Dental Bonding. Composite Resin. Acrylic resin.

\section{INTRODUÇÃO}

Como resultado da grande valorização da estética e da consciência da necessidade de tratamento ortodôntico, o número de pacientes adultos que procuram por tratamento ortodôntico aumentou consideravelmente [1,2]. Contudo, neste grupo de pacientes, pode ser encontrada maior frequência de restaurações de amálgama, de resina ou de cerâmica [1,2]. Esta situação está diretamente relacionada a problemas associados à função e à estética na dentição permanente que podem ocorrer como resultado de fratura ou de lesão cariosa extensa [3] .

A relação das demais estruturas do dente até a crista alveolar, a proporção coroa-raiz e as exigências estéticas do paciente influenciam no plano de tratamento [3]. Quando, por exemplo, um tracionamento da raiz é indicado, na maioria das vezes, um pino intrarradicular juntamente com um núcleo são utilizados para dentes tratados endodonticamente com presença de no mínimo $2 \mathrm{~mm}$ de estrutura coronária remanescente [4], e uma coroa provisória é necessária antes da extrusão da raiz [3]. A finalidade de uma coroa provisória é proteger, estabilizar e devolver função ao dente, bem como determinar o resultado estético da restauração final [3].

A Ortodontia é muitas vezes uma fase intermédia para a reabilitação oral [1,5]. Dependendo da técnica utilizada, torna-se necessário realizar colagem de bráquetes em coroas provisórias. Dessa forma existe uma grande diversidade quanto à natureza da superfície em que o bráquete será colado e isto implicará na resistência para suportar forças ortodônticas e oclusais [1,5]. Alguns estudos têm avaliado a força de união de bráquetes a superfície do esmalte utilizando compósitos resinosos ou à base de ionômero de vidro [6-8]. A adesão de bráquetes tornou-se um protocolo convencional após muitos estudos que provaram a sua eficácia, especialmente em relação à resistência ao cisalhamento [6-9].

De acordo com a literatura, os valores de resistência de união dos bráquetes em esmalte devem variar entre 5 a $8 \mathrm{MPa}[10,11]$. Por outro lado, não devem apresentar força de adesão superior, pois podem ocasionar fraturas de esmalte durante a remoção dos bráquetes $[6,8,10]$. Outros autores mencionam variações de $20 \mathrm{MPa}$ para resistência de união aceitável [12,14]. Informações relacionadas à resistência de união de bráquetes com vários materiais restauradores também estão disponíveis. Autores afirmam que é possível uma adesão eficaz entre acessórios ortodônticos e superfícies de outros materiais, além do esmalte dental, com os materiais e as técnicas disponíveis [13-16]. Para ZACHRISSON [17], a resina acrílica é recomendada para a colagem em superfícies acrílicas, pois sua força de ligação é maior do que o obtido com outros agentes de ligação. No entanto, estudos especificamente relacionados com adesão de bráquetes a restaurações provisórias de resina acrílica são bastante limitados [1-3,5]. Portanto, o objetivo deste estudo foi avaliar e comparar diferentes métodos de adesão de bráquetes metálicos colados em resina acrílica por meio da resistência ao cisalhamento. 


\section{MATERIAIS E MÉTODOS}

\subsection{Delineamento experimental}

Este estudo "in vitro" teve como objetivo avaliar a resistência ao cisalhamento da colagem de bráquetes metálicos em resina acrílica, e comparar a influência de quatros tratamentos de superfície: monômero de metilmetacrilato; monômero de metilmetacrilato associado ao cianoacrilato; condicionamento com ácido fosfórico a $37 \%$ e adesivo fotopolimerizável; condicionamento com ácido fosfórico a 37\% e adesivo fotopolimerizável associado ao cianoacrilato.

\subsection{Preparação da amostra}

Foram confeccionados 40 corpos de prova em resina acrílica (JET, Artigos Odontológicos Clássico Ltda, São Paulo, SP, Brasil). Polimetilmetacrilato (Acrílico auto polimerizante, JET, Artigos Odontológicos Clássico Ltda, São Paulo, SP, Brasil) e metilmetacrilato (JET, Artigos Odontológicos Clássico Ltda, São Paulo, SP, Brasil) foram misturados, nas proporções recomendadas pelo fabricante (uma parte de líquido para três partes de pó), em um recipiente de vidro com tampa até que a mistura atingisse a fase plástica. Em seguida, a resina foi vertida no interior de tubos cilíndricos de PVC (Polyvinyl chloride) com 3/4 de polegada e $16 \mathrm{~mm}$ de altura. Esses tubos serviram como matrizes, possibilitando a formação dos corpos de prova em resina acrílica.

\subsection{Colagem dos bráquetes}

Após a polimerização da resina, as superfícies das amostras foram homogeinizadas com lixas de carbureto de silício na ordem decrescente de abrasividade 320 e 600 (Polipan 2, São Paulo, SP, Brasil), sob refrigeração/lubrificação abundante com água corrente. Em seguida, as amostras foram polidas com pedrapomes e água. A mistura de pedra-pomes e água foi aplicada por meio de taça de borracha acoplada a baixa rotação, a uma velocidade de $500 \mathrm{rpm}$, durante um minuto, seguido de secagem com papel absorvente e jatos de ar.

A seguir, as 40 amostras foram divididas aleatoriamente em quatro grupos (G1 a G4), de acordo com o tratamento de superfície a ser utilizado para adesão dos bráquetes ortodônticos de aço inoxidável. Foi utilizado 10 corpos de prova em cada grupo [3,5].

G1 (resina acrílica) - (técnica de Nylon) monômero e polímero foram colocados separadamente em potes tipo dappen. Com auxílio de pincel, o monômero foi aplicado na região central da superfície da amostra para condicionar a região que receberia o bráquete. Em seguida, a extremidade do pincel foi umedecida com monômero e colocada em contato com polímero. As partículas de pó incorporadas pelo pincel umedecido formaram uma pequena esfera de resina que foi levada à superfície da amostra. Posteriormente, com auxílio de uma pinça ortodôntica, o bráquete foi posicionado sobre o corpo de prova em resina acrílica, mantendo-se uma pressão constante do bráquete contra o corpo de prova durante 15 segundos. Os excessos de material foram removidos com sonda exploradora $\mathrm{n}^{0} 5$.

G2 (resina acrílica e cianoacrilato) - as amostras foram confeccionadas seguindo o mesmo protocolo utilizado para o G1. Após a remoção dos excessos de resina acrílica, uma fina camada de cianoacrilato líquido (Super Bonder, Loctite, Henkel Ltda, São Paulo, SP, Brasil) foi aplicada com auxílio de ponta descartável (Cavibrush; FGM, Joinville, SC, Brasil) ao redor do bráquete.

G3 (Transbond ${ }^{\mathrm{TM}}$ XT) - foi aplicado ácido fosfórico a 37\% (Condac 37; FGM, Joinville, SC, Brasil) na região central da amostra durante 30 segundos. Em seguida, o ácido foi removido por meio de lavagem em água corrente pelo mesmo tempo. A superfície da amostra foi seca utilizando papel absorvente e jatos de ar durante 10 segundos. Adesivo (Transbond ${ }^{\mathrm{TM}}$ XT, 3M, Unitek, São José do Rio Preto, SP, Brasil) foi espalhado com pontas descartáveis (Cavibrush; FGM, Joinville, SC, Brasil) e jatos de ar foram aplicados durante 10 segundos antes da fotoativação (Emitter A, Schuster, Santa Maria, RS, Brasil) por 20 segundos. Após a realização dos preparos de superfície, foi feita a colagem dos bráquetes com a resina Transbond ${ }^{\mathrm{TM}} \mathrm{XT}$ (3M, Unitek, São José do Rio Preto, SP, Brasil). A resina Transbond ${ }^{\mathrm{TM}}$ XT foi aplicada, com o auxílio de uma espátula de inserção, em pequenas porções sobre a base dos bráquetes, sendo pressionada sobre a superfície condicionada do corpo de prova. Os excessos foram removidos com a sonda exploradora e todo o conjunto foi fotoativado por 40 segundos.

G4 (Transbond ${ }^{\mathrm{TM}}$ XT e cianoacrilato) - para este grupo foi seguido o mesmo protocolo descrito para o G3. Após polimerização do conjunto bráquete e resina, cianoacrilato líquido (Super Bonder, Loctite, Henkel Ltda, São Paulo, SP, Brasil) foi aplicado com auxílio de ponta descartável (Cavibrush; FGM, Joinville, SC, Brasil) ao redor do dispositivo metálico.

Foram utilizados 40 bráquetes metálicos, prescrição Roth, Kirium (3M/Abzil, São José do Rio Preto, SP, Brasil) designados para incisivos centrais superiores direitos, slot 022”. Baseado no estudo de RAMBHIA et al. [3], preferiu-se a utilização de bráquetes para incisivos centrais superiores, em vez de dispositivos para pré-molares. 


\subsection{Teste de cisalhamento}

Após o preparo das amostras, os ensaios de resistência ao cisalhamento foram realizados em uma máquina de ensaios universal (EMIC DL-1000, São José dos Pinhais, PR, Brasil). O equipamento foi ajustado para aplicar uma carga de 50 kgf, na intersecção da superfície da resina acrílica e margem do bráquete, a uma velocidade de $0,5 \mathrm{~mm} / \mathrm{min}$. O valor da força foi obtido no momento da ruptura da adesão. Em seguida, os valores obtidos em quilograma-força (kgf) foram registrados e convertidos em MPa por meio de cálculos específicos, levando-se em consideração a área de superfície da base do bráquete fornecida pelo fabricante $(12,89 \mathrm{~mm} 2)$.

\subsection{Análise dos dados}

Os dados foram analisados por meio de tabelas e gráficos contendo valores de média e desvio padrão.

Para verificar se os dados tinham distribuição normal foi utilizado o teste de Kolmogorov-Smirnov. Todos os grupos demonstram distribuição normal. Os dados passaram no critério de homocedasticidade avaliado pelo teste de Bartlett. Para comparar as diferenças entre os quatro grupos utilizou-se Análise de Variância (ANOVA) a um critério. Quando a ANOVA mostrou diferença estatisticamente significante foi utilizado o Teste de Tukey para as comparações múltiplas. Em todos os testes adotou-se nível de significância de $5 \%(\mathrm{p}<0,05)$.

Os testes foram executados no programa Statistica for Windows v. 12 (StatSoft Inc., USA).

\section{RESULTADOS}

A média dos valores e desvio-padrão das tensões cisalhantes (MPa) obtidos para cada grupo estão na tabela 1 e apresentação gráfica na figura 1 .

A maior média obtida a partir dos valores do teste de resistência ao cisalhamento (13,76 MPa) foi observada em G2 (resina acrílica e cianoacrilato), enquanto o menor valor (3,87 MPa) foi para o G3 (Transbond ${ }^{\mathrm{TM}} \mathrm{XT}$ ). Houve uma interação significativa entre o cianoacrilato e os materiais adesivos utilizados. Porém, quando comparados os resultados, a associação resina acrílica e cianoacrilato (G2) apresentou os maiores valores $(\mathrm{p}<0,05)$, mas não significativo em comparação aos valores obtidos para a resina acrílica utilizada isoladamente. Em relação ao poder da amostra, baseado no desvio padrão médio obtido nos quatro grupos, que foi de 4,97 MPa, adotando-se nível de significância de 5\%, a amostra de 10 elementos em cada grupo tem um poder de $80 \%$ de detectar uma diferença entre os grupos de no mínimo 7,98 MPa

Tabela 1: Valores médios e de desvio padrão da tensão de cisalhamento de adesão para cada grupo investigado (MPa).

\begin{tabular}{c|c|c}
\hline $\begin{array}{c}\text { GRUPOS } \\
\text { EXPERIMENTAIS }\end{array}$ & MÉDIA & $\begin{array}{c}\text { DESVIO- } \\
\text { PADRÃO }\end{array}$ \\
\hline $\mathrm{G} 1$ & $7,76^{\mathrm{ab}}$ & 6,96 \\
\hline $\mathrm{G} 2$ & $13,76^{\mathrm{a}}$ & 4,43 \\
\hline $\mathrm{G} 3$ & $3,87^{\mathrm{b}}$ & 4,91 \\
\hline $\mathrm{G} 4$ & $4,03^{\mathrm{b}}$ & 3,56 \\
\hline
\end{tabular}

ONEWAY ANOVA; $\mathrm{p}<0,05$ - estatisticamente significativo

Grupos com a mesma letra não possuem diferença estatisticamente significativa entre si 


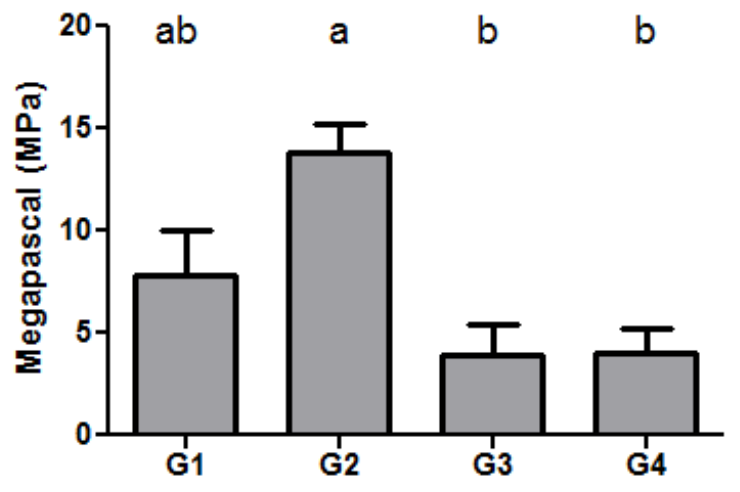

Figura 1: Média de resistência ao cisalhamento e desvios-padrão (em MPa) de todos os grupos estudados.

\section{DISCUSSÃO}

A colagem efetiva dos bráquetes continua sendo um fator de suma importância para o sucesso do tratamento ortodôntico [5] e depende de alguns fatores, tais como, a preparação da superfície, o adesivo e os materiais utilizados para a colagem, entre outros [1]. A adesão em resina acrílica não representa o ideal como proposto na literatura, acarretando assim em repetidas descolagens dos dispositivos ortodônticos e consequentemente, em problemas durante o tratamento, tais como o atraso temporal na finalização do mesmo.

Os valores médios mostrados na tabela e figura 1 são apresentadas em megapascal (MPa), os quais caracterizam a tensão provocada entre a superfície da resina acrílica e base dos bráquetes. Para obtenção desses valores, a área de base dos bráquetes e a força $(\mathrm{N})$ são levadas em consideração.

As amostras de resina acrílica foram preparadas utilizando o mesmo protocolo, assim, espera-se que o grau de rugosidade superficial seja o mesmo para todos os corpos de prova. Pequenas diferenças nas superfícies devem existir e simulam o que acontece no procedimento clínico, porém, não se prevê que influenciem nos valores de resistência de união.

As resinas acrílicas apresentam estruturas químicas de metacrilato em sua composição [3]. Assim, a ligação química é influenciada pelo número de locais reativos disponíveis sobre o material já polimerizado [3]. Uma vez que, materiais com elementos químicos semelhantes são utilizados, o poder coesivo do material é substancialmente maior do que a força de adesão obtida por adição de outro agente de ligação [1]. Entretanto, nesta pesquisa, a aplicação prévia de monômero de metilmetacrilato (G1) gerou uma resistência adesiva semelhante ao obtido após condicionamento ácido e uso do adesivo fotopolimerizável Transbond ${ }^{\mathrm{TM}}$ XT (G3).

Apesar de não se esperar que o condicionamento ácido crie porosidades na superfície da resina acrílica, o ácido proporciona uma limpeza superficial na mesma [3]. Isto, possivelmente aumenta a molhabilidade da superfície, permitindo que o adesivo fotopolimerizável Transbond ${ }^{\mathrm{TM}} \mathrm{XT}$ esteja em íntimo contato com a resina proporcionando uma ligação satisfatória [3], o que justificaria os resultados obtidos. Diferente dos resultados desta pesquisa, MASIOLI et al. [5] obtiveram maiores forças de adesão entre bráquete e provisório em resina acrílica quando a superfície foi tratada com monômero de metilmetacrilato, em comparação a superfície tratada com ácido fosfórico e adesivo fotopolimerizável Transbond ${ }^{\mathrm{TM}}$ XT.

Nos grupos em que o adesivo fotopolimerizável Transbond ${ }^{\mathrm{TM}}$ XT foi utilizado (G3 e G4), menores valores para resistência adesiva foram obtidos. Isto pode ser explicado pelo fato de que quando um material relativamente novo acaba de ser polimerizado e polido, ele apresenta um pouco mais de $50 \%$ de grupos de metacrilato não reativos. No entanto, com o passar do tempo, haverá menos grupos de metacrilato não reativos, os quais irão gerar algumas ligações cruzadas com o novo material inserido e reduzir a capacidade do monômero de penetrar na matriz. Assim, a resistência entre o material da coroa provisória e resina inserida na base do bráquete é reduzida em cerca de 50\% [18].

Cianoacrilatos são monômeros líquidos com viscosidade e aparência semelhante à da água [19]. A natureza fluida do adesivo e a tendência de agarrar aos instrumentos dificultam a sua utilização [20]. Contudo, os adesivos à base de cianoacrilato apresentam como vantagem a polimerização à temperatura ambiente, por meio de uma ligação exotérmica, que leva de 15 a 60 segundos [19,20]. Além disso, a possibilidade de "ligação instantânea" e a degradação hidrolítica permitem descolagem fácil e segura desse material. Adesivos à base de cianoacrilato têm sido bastante utilizados nas diversas áreas da Medicina e da Odontologia [20]. Alguns estudos têm mostrado o uso do cianoacrilato como adesivos cirúrgicos [19,21,22], 
durante terapia endodôntica [23,24] e para estabilização do lençol de borracha durante isolamento absoluto [25]. Os resultados clínicos e histológicos destes estudos têm demonstrado a baixa toxicidade desse material. A eficácia do cianoacrilato como adesivo ortodôntico também tem sido demonstrado [21,26-29].

Os resultados favoráveis dessas pesquisas apoiam a escolha deste material na colagem de bráquetes ortodônticos em materiais temporáreis. No presente estudo, houve maior valor de união quando a colagem dos bráquetes metálicos foi feita com monômero de metilmetacrilato e adesivo de cianoacrilato (G2). Adesivos à base de cianoacrilato podem proporcionar forças de ligação funcionais que chegam a $25 \mathrm{MPa}$, contudo variam de acordo com a superfície a ser colada e espessura da camada adesiva [28]. KRISHNAN et al. [30] constataram que os adesivos à base de cianoacrilato produziram uma força de adesão semelhante a um material adesivo à base de BIS-GMA. Para SOON et al. [28], um adesivo à base de cianoacrilato demonstrou resultados estatisticamente maiores para a resistência de união comparados aos grupos em que jateamento com óxido de alumínio e cimento resinoso foram utilizados. Outros estudos compararam ainda a resistência de união do adesivo à base de cianoacrilato em esmalte com adesivos convencionais [31,32]. Os resultados obtidos indicaram valores para força clinicamente aceitáveis. Além disso, não houve diferença significativa para os valores obtidos no teste de cisalhamento entre os materiais. Embora as pesquisas demonstrem excelentes resultados para força de união de adesivos à base cianoacrilato, alguns estudos investigam o efeito deste material em meio úmido [20,27].

Embora valores elevados para a resistência de união de bráquetes em esmalte não seja desejado, a resistência adesiva de bráquetes aos materiais temporários deve ser forte o suficiente para resistir às forças mastigatórias sem acarretar inúmeras descolagens [1,33]. De acordo com REYNOLDS [10] e WHITLOCK et al. [11], a mínima resistência adesiva clinicamente aceitável varia entre 5 e 8 MPa. Nos grupos avaliados, os valores das médias de resistência de união indicam que o uso do monômero de metilmetacrilato associado ou não ao cianoacrilato podem ser utilizados clinicamente para adesão de bráquetes metálicos à superfície de resina acrílica, visando a permanência do acessório durante o tratamento ortodôntico, minimizando o descolamento. No entanto, é importante considerar que existe uma influência da degradação intraoral da resistência adesiva [34,35] causada por fadiga [36], temperatura, variações de pH [37] e degradação microbiológica [38]. Além disso, resina acrílica ou composta mantém uma força de ligação acima do nível aceitável para utilização clínica [10,11].

\section{CONCLUSÃO}

De acordo com a metodologia empregada no presente estudo, o tratamento de superfície e agente adesivo tem um efeito significativo na resistência de união dos bráquetes fixados aos materiais provisórios. Contudo, o uso do cianoacrilato não proporcionou maior resistência de adesão do bráquete à resina acrílica quando comparado à utilização dos materiais de forma isolada avaliado estatisticamente. O resultado superior quanto à força de adesão do grupo G2 (resina acrílica e cianoacrilato), nos possibilita sugerir sua utilização na prática clínica.

\section{AGRADECIMENTOS}

Os autores agradecem à FAPITEC/SE (Fundação de Apoio à Pesquisa e Inovação Tecnológica do Estado de Sergipe) e a CAPES (Coordenação de Aperfeiçoamento de Pessoal de Nível Superior) pelos subsídios obtidos para a realização deste trabalho por meio do PROMOB.

\section{BIBLIOGRAFIA}

[1] ALMEIDA, J.X., DEPRÁ, M.B., MARQUEZAN, M., et al., "Effects of surface treatment of provisional crowns on the shear bond strength of brackets”, Dental Press J Orthod, v. 18, n. 4, pp. 29-34, July-Aug. 2013.

[2] RIBEIRO, A.A., MORAIS, A.V., BRUNETTO, D.P., et al., "Comparison of shear bond strength of orthodontics brackets on composite resin restorations with different surface treatments", Dental Press $J$ Orthod., v. 18, n. 4, pp. 98-103, July-Aug. 2013.

[3] RAMBHIA S., HESHMATI, R., DHURU, V., et al., "Shear Bond Strength of Orthodontic Brackets Bonded to Provisional Crown Materials Utilizing Two Different Adhesives”, Angle Orthodontist., v. 79, n. 4, pp. 784-89. 2009.

[4] TRUSHKOWSKY, R.D., 'Restoration of endodontically treated teeth: criteria and technique considerations”, Quintessence Int., v. 45, n. 7, pp. 557-67, Jul-Aug. 2014.

[5] MASIOLI, D.L.C., ALMEIDA, M.A.O., MASIOLI, M.A., et al., "Avaliação do efeito de tratamentos superficiais sobre a força de adesão de bráquetes em provisórios de resina acrílica”, Dental Press J Orthod, v. 16, n. 1, pp. 37-47, Jan-Feb. 2011. 
[6] POWERS, J.M., KIM, H.B., TURNER, D.S., “Orthodontic adhesives and bond strength testing”, Semin Orthod., v. 3, n. 3, pp. 147-56, Sep. 1997.

[7] SINHA, P.K., NANDA, R.S., DUNCANSON, M.G., et al., "Bond strengths and remnant adhesive resin on debonding for orthodontic bonding technique”, Am J Orthod Dentofacial Orthop., v. 108, n. 3, pp. 302-7. 1995.

[8] TRIMPENEERS, L.M., VERBEECK, R.M., DERMAUT, L.R., et al., “Comparative shear bond strength of some orthodontic bonding resins to enamel”, Eur J Orthod., v. 18, n. 1, pp. 89-95. 1996.

[9] WANG, W.N., LI, C.H., CHOU, T.H., et al., "Bond strength of various bracket base designs", Am J Orthod Dentofacial Orthop., v. 125, n. 1, pp. 65-70. 2004.

[10] REYNOLDS, I.R., FRAUNHOFER, J.A.V., "Direct bonding of orthodontic attachments to teeth: the relation of adhesive bond strength to gauze mesh size”, Br J Orthod., v. 3, n. 2, pp. 91-5. 1976.

[11] WHITLOCK, B.O., EICK, J.D., ACKERMAN, R.J.Jr., et al., "Shear strength of ceramic brackets bonded to porcelain”, Am J Orthod Dentofacial Orthop, v. 106, n. 4, pp. 358-64. 1994.

[12] BRADBURN, G., PENDER, N., "An in vitro study of the bond strength of two lightcured composites used in the direct bonding of orthodontic brackets to molars". Am J Orthod Dentofacial Orthop., v. 102, n. 5, pp. 418-26. 1992.

[13] ZACHRISSON, B.U., "Orthodontic bonding to artificial tooth surfaces: clinical versus laboratory findings”, Am J Orthod Dentofacial Orthop., v. 117, n. 5, pp. 592-4, May.2000.

[14] WOOD, D.P., JORDAN, R.E., WAY, D.C., GALIL, K.A., "Bonding to porcelain and gold”, Am J Orthod., v. 89, n. 3, pp. 194-205, Mar. 1986.

[15] BERRY, N.G., D'ALMEIDA, J.R.M., "The influence of circular centered defects on the performance of carbon-epoxy single lap joints”, Polym Testing, v. 21, n. 4, pp. 373-9. 2002.

[16] NEWMAN, G.V., "Bonding to porcelain”, J Clin Orthod., v. 17, pp. 53-5. 1993.

[17] ZACHRISSON, B.U., "Bonding in orthodontics", In: Graber TM, Swain BF, Orthodontic current and principles and techniques, St. Louis: C.V. Mosby, pp. 485-563, 1985.

[18] GEGAUFF, A.G., WILKERSON, J.J., "Fracture toughness testing of visible lightand chemical-initiated provisional restoration resins”, Int J Prosthodont, v. 8, n. 1, pp. 62-8. 1995.

[19] NEGRI, M.R., PANZARINI, S.R., POI, W.R., et al., "Use of a cyanoacrylate ester adhesive for splinting of replanted teeth”, Dental Traumatology, v. 24, pp. 695-7. 2008.

[20] AL-MUNAJED, M.K., GORDON, P.H., MCCABE, J.F., "The Use of a Cyanoacrylate Adhesive for Bonding Orthodontic Brackets: an ex-vivo study”, Journal of Orthodontics, v. 27, pp. 255-60. 2000.

[21] PEREZ, M., FERNANDEZ, I., MARQUEZ, D., BRETANA, R.M., "Use of N-butyl-2-cyanoacrylate in oral surgery: biological and clinical evaluation”, Artif Organs, v. 24, pp. 241-3. 2000.

[22] KULKARNI, S., DODWAD, V., CHAVA, V., "Healing of periodontal flaps when closed with silk sutures and N-butyl cyanoacrylate: a clinical and histological study”, Indian J Dent Res, v. 28, pp. 72-7. 2007.

[23] WINIK, R., ARAKI, A.T., NEGRÃO, J.A., et al., "Sealer penetration and marginal permeability after apicoectomy varying retrocavity preparation and retrofilling material”, Braz Dent J, v. 17, pp. 323-7. 2006. [24] ALBURQUERQUE, D.S., GOMINHO, L.F., SANTOS, R.A., "Histologic evaluation of pulpotomy performed with ethylcyanoacrylate and calcium hydroxide”, Braz Oral Res, v. 20, pp. 226-30. 2006.

[25] SOUSA-NETO, M.D., SAQUY, P.C., SILVA-SOUSA, Y.T.C., et al., "Rubber dam isolation of the operative field with the aid of cyanoacrylates: case report”, Rev Bras Odont, v. 53, pp. 36-7. 1996.

[26] CRABB, J.J., WILSON, H.J., "Use of some adhesives in orthodontics”, Dental Practitioner, v. 22, pp. 111-2. 1971.

[27] HOWELLS, D.J., JONES, P., "In vitro evaluation of a cyanoacrylate bonding agent”, British Journal of Orthodontics., v. 16, pp. 75-8. 1989.

[28] SOON, H.I., GILL, D.S., JONES, S.P., "A study to investigate the bond strengths of orthodontic brackets bonded to prosthetic acrylic teeth”, J Orthod, v. 6, 1465313315Y0000000003. 2015.

[29] MANFRIN, T.M., POI, W.R., MENDONÇA, M.R., et al., "Analysis in vitro of direct bonding system with cyanoacrylate ester and orthodontic wires”, Dental Traumatology, v. 25, pp. 229-32. 2009.

[30] KRISHNAN, P.H., GUPTA, D.S., SHARMA, V.P., “Application of cyanoacrylate in orthodontics”, Journal of Polymer Materials, v. 11, pp. 299-300. 1994.

[31] BISHARA, S.E., VONWALD, L., LAFFON, J.F., et al., "Effect of using a new cyanoacrylate adhesive on the shear bond strength of orthodontic brackets", Angle Orthod, v. 71, pp. 466-9. 2001.

[32] BISHARA, S.E., LAFFON, J.F., VONWALD, L., WARREN, J.J., "Effect of time on the shear bond strength of cyanoacrylate and composite orthodontic adhesives", Am J Orthod Dentofacial Orthop, v. 121, pp. 297-300. 2002. 
[33] AL JABBARI, Y.S., AL TAWEEL, S.M., AL RIFAIY, M., ALQAHTANI, M.Q., KOUTSOUKIS, T., ZINELIS, S., "Effects of surface treatment and artificial aging on the shear bond strength of orthodontic brackets bonded to four different provisional restorations”, Angle Orthodontist, v. 84, n. 4, pp. 649-55. 2014. [34] ELIADES, T., BRANTLEY, W.A., "The inappropriateness of conventional orthodontic bond strength assessment protocols”, Eur J Orthod, v. 22, pp. 13-23. 2000.

[35] ELIADES, T., KATSAVRIAS, E., ZINELIS, S., ELIADES, G., “Effect of loading rate on bond strength. J Orofac Orthop, v. 65, pp. 336-42. 2004.

[36] MOSELEY, H.C., HORROCKS, E.N., PEARSON, G.J., DAVIES, E.H., "Effects of cyclic stressing on attachment bond strength using glass ionomer cement and composite resin”, Br J Orthod, v. 22, pp. 237.1995 .

[37] KANDIL, S.H., KAMAR, A.A., SHAABAN, S.A., TAYMOUR, S.A., MORSI, S.E., “Effect Of temperature and ageing on the mechanical properties of dental polymeric composite materials", Biomaterials, v. 10, pp. 540-4. 1989.

[38] MATASA, C.G., "Microbial attack of orthodontic adhesives”, Am J Orthod Dentofacial Orthop, v. 108, pp. 132-41. 1995. 